\title{
Type B Aortic Dissection Repair Using a Thoraflex Hybrid Prosthesis in a Complex Aortic Arch Anatomy
}

\author{
Moussa Abi Ghanem, MD ${ }^{1 *}$, Mario Gomez-Sanchez, MD ${ }^{1}$, Xavier Chaufour, MD, PhD², \\ Bertrand Marcheix, MD, PhD ${ }^{1}$ \\ ${ }^{1}$ Department of Cardiovascular Surgery, Centre Hospitalier Universitaire Rangueil, Toulouse, France \\ 2 Department of Vascular Surgery, Centre Hospitalier Universitaire Rangueil, Toulouse, France
}

\begin{abstract}
Thoracic endovascular aortic repair (TEVAR) is recognized as an attractive option to treat complicated Type B aortic dissection. Nevertheless, TEVAR is not always technically possible. We report the case of a 53-year-old male with complicated Type B aortic dissection, in the setting of a complex anomalous aortic arch anatomy with an aneurysmal aberrant right subclavian artery. He was successfully treated by the frozen elephant trunk technique using the Thoraflex hybrid graft.

Copyright $\odot 2016$ Science International Corp.
\end{abstract}

\section{Key Words \\ Bovine arch • Dissection • Aorta}

\section{Introduction}

Extensive aortic pathologies involving the aortic arch and the proximal descending aorta remain challenging for cardiac surgeons. After the introduction of the frozen elephant trunk technique in 1996 [1], several types of prosthesis have been developed to repair this combined disease of the aortic arch and the proximal descending aorta.
Thoraflex, the hybrid prosthesis of Vascutek (Scotland-UK), is a new commercially available prosthesis. It is composed of a distal endograft and a proximal 4-branched Gelweave graft, with an incorporated sewing collar in between [2].

\section{Case Presentation}

A 53-year-old man, with past history of hypertension, dyslipidemia, Type 2 diabetes, obesity (body mass index 40), sleep apnea, and smoking, was admitted to our service for surgical management of a complicated Type B aortic dissection.

He presented first to the emergency room with a sudden onset of chest pain. His chest computed tomography (CT) angiogram showed a Type B aortic dissection with a complex anomalous aortic arch anatomy. His first aortic arch branch is a common trunk dividing into the right common carotid artery and the left common carotid artery (truncus bicaroticus). The second aortic arch branch is the left subclavian artery, followed by a large aneurysmal aberrant right subclavian artery running a retroesophageal course (arteria lusoria). The descending aortic dissection starts at the level of the arteria lusoria and extends to the renal arteries with both the celiac trunk and the superior

\footnotetext{
* Corresponding Author:

Moussa Abi Ghanem, MD

Department of Cardiovascular Surgery,

Centre Hospitalier Universitaire Rangueil

1, Avenue du Pr Jean Poulhès, 31509 Toulouse, France

Tel.: +33 7 53106803; Fax: +33 5 61322959; E-Mail: moussaabighanem@hotmail.com
} 
mesenteric artery perfused by the false lumen, without any clinical or radiological signs of organ malperfusion. The main port of entry for the dissection into the false lumen was at the level of the arteria lusoria, 1 $\mathrm{cm}$ distal to the origin of the left subclavian artery, with multiple additional ports of entry along the rest of the descending aorta (Figure 1A and B).

His transthoracic cardiac ultrasound showed a normal left ventricular ejection fraction (60\%). His coronary angiogram showed no significant coronary stenosis.

Medical treatment was initiated and another CT scan was performed one week later because of recurrence of chest pain. That CT scan demonstrated a complicated subacute Type B aortic dissection with rapid enlargement of the maximal aortic diameter $(>1 \mathrm{~cm}$ in 1 week) at the level of the proximal third of the descending aorta.

After a multidisciplinary discussion, thoracic endovascular aortic repair (TEVAR) was discussed but not retained as a reasonable option, considering the complex, aberrant anatomy of this patient. A two-stage surgical treatment was chosen. The first stage consisting of a right carotid to right subclavian bypass, with occlusion of the arteria lusoria using an intravascular plug. The second stage consisting of a complete arch replacement and an antegrade deployment of a
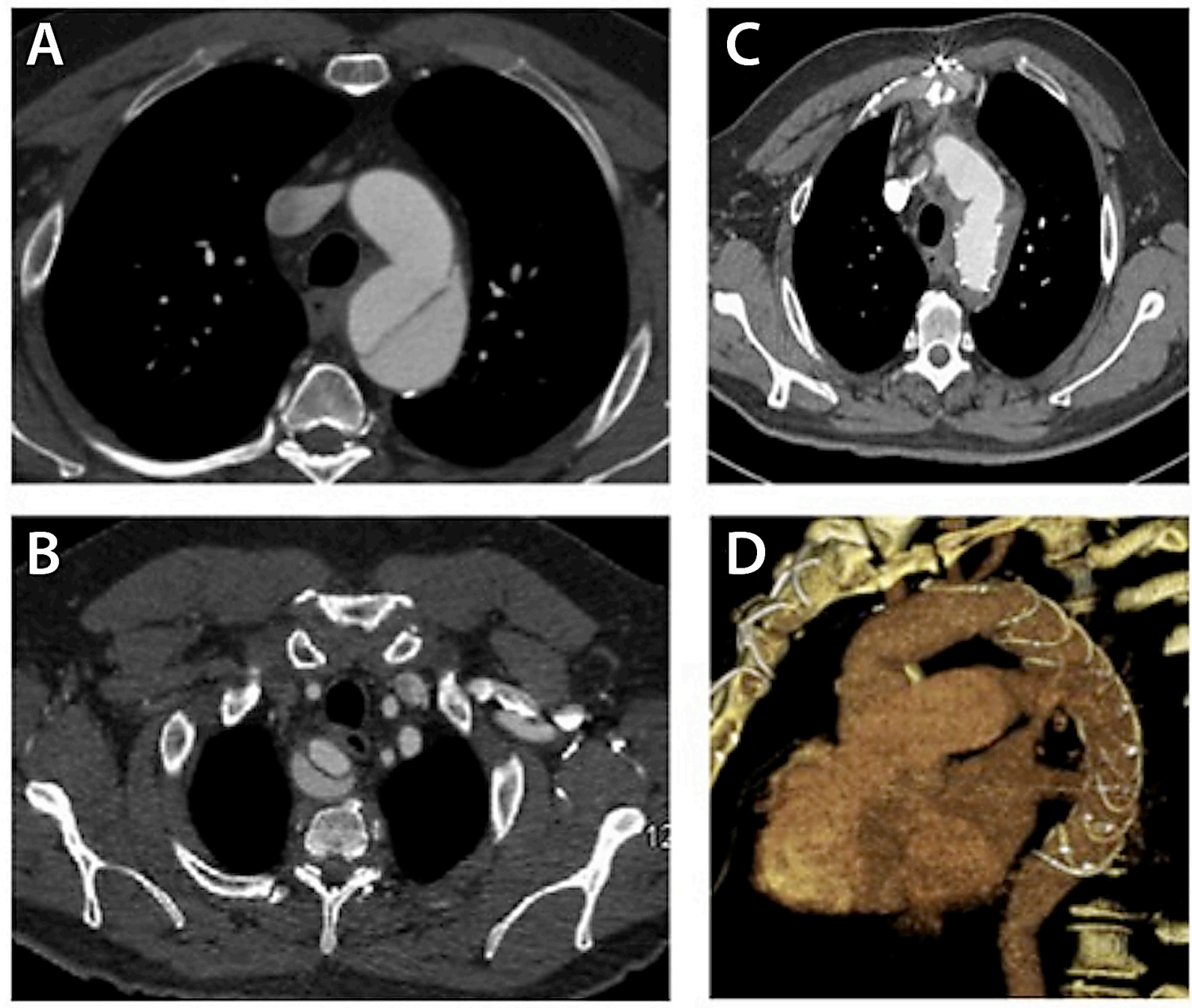

Figure 1. Panel A. Type B aortic dissection starting at the level of the aortic isthmus. Panel B. Aberrant right subclavian artery crossing behind the esophagus with retrograde dissection in the aberrant artery. Panels C and D. Three-month postoperative CT scan. Note: Thoraflex prosthesis in place with no leaks, no false aneurysm, and a thrombosed false lumen down to the level of the diaphragm. 
stented endograft in the descending aorta using the Thoraflex hybrid graft (Figure 2).

The first surgery consisted of a right carotidsubclavian bypass with an $8 \mathrm{~mm}$ Dacron prosthesis with insertion of an occlusive plug (PLUG 2 Amplatze $16 \mathrm{~mm}$ ) into the aberrant right subclavian artery. The plug was delivered through the right brachial artery in a retrograde fashion.

Six weeks after this successful bypass, the patient was readmitted for surgical management of his aneurysmal dissection. The hybrid Thoraflex prosthesis was inserted using the frozen elephant trunk technique.

This second stage surgery was performed under cardiopulmonary bypass (CPB) with right femoral artery cannulation, moderate hypothermia, short hypothermic circulatory arrest ( $8 \mathrm{~min}$ ) and selective anterograde cerebral perfusion into both carotid arteries (61 min). Myocardial protection was achieved by continuous retrograde infusion of cold blood into the coronary sinus.

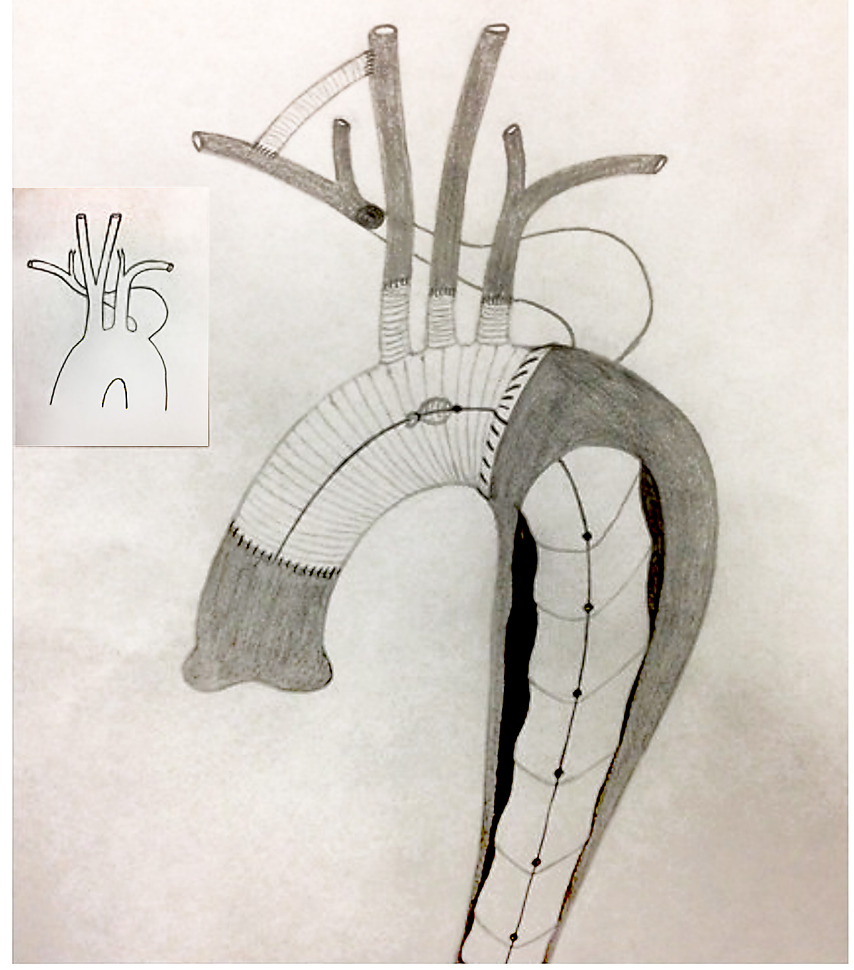

Figure 2. End result figure showing reconstruction of the aortic arch using the Thoraflex prosthesis with the right carotidsubclavian bypass graft in place. (In the left upper box is depicted the original complex aortic arch anatomy, with arteria lusoria and truncus bicaroticus.)
The endovascular part of the Thoraflex device was first deployed into the open true lumen of the descending aorta. Then, the distal anastomosis was performed with interrupted sutures proximal to the origin of the aberrant right subclavian artery, using the sewing collar that separates the distal endograft from the proximal 4-branched graft. This sewing collar contains many folds that disappear when the stent is completely deployed at $37^{\circ} \mathrm{C}$; that is why in moderate hypothermia when the deployment is still incomplete, the collar folds makes interrupted sutures much easier than running sutures.

The supra-aortic trunks were reimplanted separately in the three ad hoc branches, after which CPB was antegradely reinitiated via the fourth branch. Finally, the proximal anastomosis was performed at the level of the sino-tubular junction by continuous suture of Prolene 4-0 reinforced by circumferential Teflon felt. The patient was rewarmed during the antegrade reperfusion and CPB was easily discontinued after 167 minutes.

The postoperative course was uneventful. The patient was discharged home on postoperative day 7 after a good postoperative control CT scan.

Three months later, the patient continues to do well. Another control CT angiogram showed no leaks or false aneurysms, demonstrating a thrombosed false lumen (Figure $1 C$ and D).

\section{Discussion}

Ateria lusoria is a well-known common congenital vascular anomaly of the aortic arch, with an incidence ranging between $0.5 \%$ and $2.5 \%$ [3]. Truncus bicaroticus, a bovine aortic arch variant in humans, is another less frequent aortic arch anomaly, with a reported prevalence of less than $0.1 \%$ [4]. The coexistence of an arteria lusoria with a truncus bicaroticus represents an extremely rare combined anomaly of the aortic arch. Very few cases were reported in English literature.

TEVAR is recognized as an attractive option for complicated Type B aortic dissections [5]. Nevertheless TEVAR is not always technically feasible. When the anatomy of the dissection is not favorable for TEVAR, for example in cases with an acute angled aortic arch, entry tear close to the left subclavian 
artery, and entry tear in the concavity of the distal arch, the risk of periprocedural failure is elevated [6]. In these cases, open surgical treatment might be considered as a better approach. In our patient endografting was contraindicated, because there was not enough proximal landing zone between the port of entry, the left subclavian artery and the arteria lusoria.

In 1983, Borst [7] introduced the elephant trunk procedure as a new technique for aortic arch surgery. It was divided into two stages. The first stage consists of the prosthetic replacement of the ascending aorta and the aortic arch with an additional elephant trunklike extension of the arch graft into the descending aorta. The second stage consists of using this elephant trunk-like extension to replace the descending aorta through a lateral thoracic or thoraco-abdominal approach [8]. This two-stage surgery was associated with a high morbidity and a significant mortality, with substantial cumulative risk of the two major surgical procedures and frequent failure to complete the second stage.

In 1996, the frozen elephant trunk (FET) technique, a hybrid repair combining endovascular treatment with conventional surgery, was introduced to simplify aortic arch surgery. It allows concomitant repair of the aortic arch and the proximal descending aortic aneurysms in a single stage approach. FET is considered an interesting approach for complicated Type B aortic dissections when TEVAR is not possible and in patients who are good candidates for open surgery [6]. This technique can offer as well a better landing zone for a secondary TEVAR procedure later on, in the descending aorta if necessary [9].

At the present time, multiple FET prostheses have been developed, such as the custom-made Chavan-Haverich (Curative $\mathrm{GmbH}$, Dresden,
Germany), the Jotec E-vita (Jotec $\mathrm{GmbH}$, Hechingen, Germany) and the Thoraflex (Vascutek, Terumo, Inchinnan, Scotland, United Kingdom) prosthesis.

In March 2014, Leone et al. [10] published a case report of two patients with similar complex aortic arch disease (aberrant right subclavian artery) treated with the frozen elephant trunk technique using the Jotec E-vita prosthesis. The first patient had an ascending aortic aneurysm extending to his distal arch while the second one was diagnosed with a chronic Type B dissection.

To our knowledge, this is the first case report of Type B aortic dissection with an arteria lusoria and a truncus bicaroticus treated with the Thoraflex hybrid prosthesis.

In conclusion, we can say that hybrid repair (ascending and arch replacement combined with stenting of the descending aorta) represents a valuable tool for the treatment and simultaneous reconstruction of the aortic arch and the proximal descending aorta. Such an approach permits one-stage treatment of complex aortic pathologies.

Despite the significant morbidity and mortality (especially paraplegia), FET is becoming an indispensable tool. Studies and follow up will be needed to evaluate the medium and long term results of this procedure as well as to refine the indications and the limitations.

\section{Conflict of Interest}

The authors have no conflicts of interest relevant to this publication.

\section{Comment on this Article or Ask a Question}

\section{References}

1. Kato M, Ohnishi K, Kaneko M, Ueda T, Kishi D, Mizushima T, Matsuda H. New graft-implanting method for thoracic aortic aneurysm or dissection with a stented graft. Circulation. 1996;94:II188-193. PMID: 8901744

2. Shrestha M, Pichlmaier M, Martens A, Hagl C, Khaladj N, Haverich A. Total aortic arch replacement with a novel four-branched frozen elephant trunk graft: First-in-man results.
Eur J Cardiothorac Surg. 2013;43:406-410. DOI: $10.1093 /$ ejcts/ezs296

3. Myers PO, Fasel JH, Kalangos A, Gailloud P. Arteria lusoria: Developmental anatomy, clinical, radiological and surgical aspects. Ann Cardiol Angeiol. 2010;59:147-154. DOI: 10.1016/j.ancard.2009.07.008

4. Ozates M, Nazaroglu H, Uyar A. MR angiography in diagnosis of aberrant right subclavian artery associated with common carotid trunk. Eur Radiol. 2000;10:1503. DOI: 10.1007/s003300000335

5. Fattori R, Tsai T, Myrmel T, Evangelista A, Cooper J, Trimarchi S, et al. Complicated acute type $B$ dissection: Is surgery still the best option? JACC: Cardiovasc Interv. 2008; 395-402. DOI: 10.1016/j.jcin.2008.04.009

6. Weiss G, Wolner I, Folkmann S, Sodeck G, Schmidli J, Grabenwoger M, et al. The location of the primary entry tear in acute type $B$ 
aortic dissection affects early outcome. Eur J Cardiothorac Surg. 2012;42:571576. 10.1093/ejcts/ezs056

7. Borst HG, Walterbusch G, Schaps D. Extensive aortic replacement using "elephant trunk" prosthesis. Thorac Cardiovasc Surg. 1983;31:37-40. DOI: 10.1055/s-2007-1020290

8. Pichlmaier MA, Teebken OE, Baraki $\mathrm{H}$, Haverich A. The frozen elephant trunk technique. Multimed Man Cardiothorac Surg. 2007;2007:mmcts.2006.001990. DOI: $10.1510 /$ mmcts.2006.001990
9. Weiss $G$, Tsagakisb $K$, Jakobb $H$, Di Bartolomeoc R, Pacinic D, Barberioc G, et al. The frozen elephant trunk technique for the treatment of complicated type B aortic dissection with involvement of the aortic arch: multicentre early experience. Eur J Cardiothorac Surg. 2015;47:106-114. DOI: 10.1093/ejcts/ezu067

10. Leone A, Pantaleo A, Pacini D, Di Marco L, Di Bartolomeo R. Single-stage frozen elephant trunk reconstruction in complex disease of the aortic arch with aberrant right subclavian artery. J Card Surg. 2014;29: 234-237. DOI: $10.1111 /$ jocs.12257

Cite this article as: Abi Ghanem $M$, Gomez-Sanchez M, Chaufour X, Marcheix B. Type B Aortic Dissection Repair Using a Thoraflex Hybrid Prosthesis in a Complex Aortic Arch Anatomy. AORTA (Stamford) 2016;4(1):11-15. DOI: http://dx.doi.org/10.12945/j.aorta. 2015.15.017 